\section{ONOMÁVADIN}

Revista semestral de lingüística, filología y traducción
PONTIFICIA UNIVERSIDAD CATÓLICA DE CHILE FACULTAD DE LETRAS

\title{
Descripción fonético-acústica de la sexta vocal en el chedungun hablado en Alto Bío-Bío ${ }^{1}$
}

Acoustic-phonetic description of the sixth vowel of the spoken chedungun in Alto Bío-Bío.

\section{Jaime Soto-Barba \\ Universidad de Concepción \\ Chile}

\section{Isolina Lara}

Universidad de Concepción

Chile

\section{Gastón Salamanca}

Universidad de Concepción

Chile

\section{(c) $($ i $\fallingdotseq$}

Jaime Soto-Barba: Departamento de Español, Facultad de Humanidades y Arte, Universidad de Concepcion, Chile. I Correo electrónico: jstot@udec.cl

Isolina Lara: Departamento de Español, Facultad de Humanidades y Arte, Universidad de Concepcion, Chile. | Correo electrónico: isolinalara@gmail.com

Gastón Salamanca: Departamento de Español, Facultad de Humanidades y Arte, Universidad de Concepcion, Chile. I Correo electrónico: gsalaman@udec.cl 


\section{Resumen}

Este es un trabajo de carácter descriptivo cuyo objetivo es presentar los principales resultados de un análisis fonético-acústico de los formantes 1 y 2 ( $F 1$ y F2) de las realizaciones tónicas de la denominada sexta vocal del chedungun, un dialecto del mapudungun, en dos sectores de la comuna Alto Bío-Bío (Queuco y Bío-Bío), en 10 informantes masculinos que produjeron estos sonidos, a partir de más de 100 ítems léxicos pre- parados especialmente para la observación del sistema fonético-fonológico del chedungun. En los datos analizados, los resultados de este estudio demuestran que, por una parte, no se manifiesta incidencia de la procedencia geográfica en la producción de [ə] y [u] y, por otra, que la gran mayoría de las producciones fonéticas se realizaron como [ə], en comparación con [u].

Palabras clave: chedungun; fonética acústica; F1 y F2; sexta vocal.

\section{Abstract}

This study presents the main results obtained from a phonetic-acoustic analysis of formants 1 and 2 (F1\& F2) of the tonic production of the sixth vowel from chedungun, a dialect of mapudungun, in two locations of Alto Bío-Bío (Queuco and Bío-Bío). These sounds were produced by 10 male speakers from a corpus of more than 100 lexical items specially selected to examine the chedungun phonetic-phonologic system. The data and results on this study indicate by one hand that there is not a significant geographical influence in the production of [ə] and [u], and also that most of the phonetic production were performed as [ə] instead of [u].

Keywords: chedungun; phonetic acoustic; F1 and F2; sixth vowel. 


\section{Introducción}

Este trabajo presenta los principales resultados de una investigación descriptiva en la cual se analizaron espectrográficamente en el chedungun, un dialecto del mapudungun hablado en la comuna de Alto Bío-Bío, las ocurrencias fonéticas de la denominada sexta vocal, cuyas realizaciones representamos con [ə] y [u], y que corresponden, en IPA, a Ios fonos medio central no redondeado y alto posterior no redondeado, respectivamente. Los objetivos de este trabajo son describir el comportamiento fonético-acústico del primer y segundo formante (F1 y F2) de las vocales tónicas del chedungun y determinar si se manifiesta o no variación de los formantes vocálicos, considerando dos zonas geográficas de la comuna, esto es, Queuco y Bío-Bío. Asimismo, al establecer comparaciones entre los resultados de esta investigación y otros en los que se han utilizado procedimientos espectrográficos, se busca aportar a la discusión de uno de los tópicos relevantes del chedungun, a saber, la forma básica fonológica de la sexta vocal de este dialecto del mapudungun.

\subsection{Estudios fonético-fonológicos pro- minentes sobre la variación alofónica de la sexta vocal del mapudungun \\ 1.1.1. Sobre los estudios en el mapudun- gun en general}

Aunque ya los primeros misioneros que se asentaron en la Araucanía reportaron la existencia de un sexto fonema vocálico en el mapuche - la lla- mada sexta vocal- (Valdivia, 1606; Febrés, 1765; Havestadt, 1777; Augusta, 1903, entre otros), las primeras apreciaciones que se avienen con un acercamiento desde la disciplina lingüística se encuentran en la monumental obra de Rodolfo Lenz (1905-1910).

Sin embargo, es con Jorge Suárez (1959) que la presentación de las descripciones de esta lengua se comienza a realizar de acuerdo con el metalenguaje que caracteriza al nivel fonéticofonológico. De ahí en adelante, es posible apreciar una cantidad no menor de descripciones en las cuales se presenta, entre otros, este fonema del mapuche con sus correspondientes realizaciones alofónicas y la distribución contextual de estas.

Un buen resumen del derrotero histórico que ha seguido el tratamiento de la sexta vocal -al que cabría agregar, no obstante, el trabajo de Álvarez-Santullano (1986) - es el que nos presentan Dehnhardt, Valenzuela y Villarroel (2015) y que reproducimos aquí (véase la figura 1 ).

\subsubsection{Sobre los estudios en el chedun- gun hablado en Alto Bío-Bío, en particu- lar}

Con respecto a la variante dialectal que se aborda en este artículo -el habla adulta del chedungun hablado en Alto Bío-Bío-, se reportan los trabajos de Sánchez (1989) y Salamanca (1997). Ambos autores coinciden en distinguir seis fonemas vocálicos y no reportar la presencia del fono [J]; difieren, sin embargo, en que Salamanca asigna estatus fonémico a [t], [n] [1] y [y], mien-

\section{FIGURA 1}

Tabla de Dehnhardt, Valenzuela y Villarroel (2015), que muestra los estudios clásicos que incorporan la alofonía de la sexta vocal y su distribución

\begin{tabular}{|c|c|c|c|}
\hline AUTOR & FONEMA & ALÓFONOS & DESCRIPCIÓN \\
\hline \multirow{2}{*}{ Suárez (1959) } & \multirow{2}{*}{ lì) } & [ï] & Acentuación: tónica \\
\hline & & [ə] & Acentuación: átona \\
\hline
\end{tabular}




\begin{tabular}{|c|c|c|c|}
\hline \multirow{2}{*}{ Echeverría y Contreras (1965) } & \multirow{2}{*}{ /ì/ } & [ï] & $\begin{array}{l}\text { Acentuación: tónica } \\
\text { Posición lengua: alta y posterior. }\end{array}$ \\
\hline & & [ə] & $\begin{array}{l}\text { Acentuación: átona. } \\
\text { Posición lengua: central y media. }\end{array}$ \\
\hline \multirow[b]{2}{*}{ Salas (1978 y 1992) } & \multirow{2}{*}{$\begin{array}{l}\text { 1978: /ï/ } \\
\text { 1992: /ü/ }\end{array}$} & $\begin{array}{l}\text { 1978: [ї] } \\
\text { 1992: [ü] }\end{array}$ & $\begin{array}{l}\text { Posición lengua: alta y posterior. } \\
\text { Posición silábica: inicial, interna y final. }\end{array}$ \\
\hline & & [ə] & $\begin{array}{l}\text { Posición lengua: media y central. } \\
\text { Posición silábica: interna (entre } \\
\text { consonantes) y final. }\end{array}$ \\
\hline Lagos (2001) & /ì/ & [ï] & $\begin{array}{l}\text { Posición lengua: alta y posterior. } \\
\text { Posición silábica: inicial y final. }\end{array}$ \\
\hline \multirow{2}{*}{ Zúñiga (2006) } & \multirow{2}{*}{ /ü/ } & [i] & Posición silábica: inicial e interna. \\
\hline & & {$[ə]$} & Posición silábica: interna y final. \\
\hline \multirow{2}{*}{ Smeets (2008) } & \multirow{2}{*}{ /ü/ } & {$[\ddot{i}]$} & $\begin{array}{l}\text { Acentuación: tónica } \\
\text { Posición lengua: alta }\end{array}$ \\
\hline & & [ə] & $\begin{array}{l}\text { Acentuación: átona } \\
\text { Posición lengua: media }\end{array}$ \\
\hline \multirow{2}{*}{ Salamanca y otros (2009) } & \multirow{2}{*}{ /ï/ } & [ï] & $\begin{array}{l}\text { Posición lengua: alta y posterior. } \\
\text { Posición silábica: inicial. }\end{array}$ \\
\hline & & [ə] & $\begin{array}{l}\text { Posición lengua: media y central. } \\
\text { Posición silábica: interna y final. }\end{array}$ \\
\hline \multirow[t]{2}{*}{ Sadowsky y otros (2013) } & /९/ & [э] & $\begin{array}{l}\text { Acentuación: tónica } \\
\text { Posición lengua: media cerrada y } \\
\text { central. }\end{array}$ \\
\hline & & [i] & $\begin{array}{l}\text { Acentuación: átona } \\
\text { Posición lengua: media más cerrada } \\
\text { que [э] y central. }\end{array}$ \\
\hline
\end{tabular}

tras que Sánchez interpreta las interdentales como alófonos de las alveolares / $t, n, l /$, respectivamente, y el fono [y] como un apoyo de la sexta vocal.

Ahora bien, en relación con la temática que nos ocupa - el vocalismo del chedungun hablado en Alto Bío-Bío, en general, y el estatus de la sexta vocal, en particular-, en su trabajo del año 1997, Salamanca reporta las variantes [u] ([ü], en su transcripción) y [ə], indicando que este último fono ocurre "en todos los contextos, excepto en posición inicial. En posición final, variación libre con [ü]” (Salamanca, 1997: 115). Así, la ocurrencia de [ə] es descrita como mayoritaria con respecto a [u]; sin embargo, siguiendo una tendencia de los reportes supra-dialectales del mapudungun (o pan-mapudungun), selecciona la variante posterior como forma representativa del fonema.

En el artículo publicado en 2012 por Henríquez y Salamanca, en cambio, ya se releva la prominencia del fono [ə] en esta zona, y se lo caracteriza como representativo del fonema, aunque también se reconocen algunas realizaciones alofónicas [u] en la población escolar estudiada.

\subsubsection{Sobre los estudios en el mapudun- gun con procedimientos fonético-acús- ticos}

Los estudios de las vocales del mapudungun desde una perspectiva fonético-acústica son todavía 
muy escasos, y, específicamente, los vinculados al chedungun, inéditos. Aún así, una revisión exhaustiva de trabajos realizados con procedimientos espectrográficos de las vocales de la lengua que nos ocupa muestra la existencia de 4 estudios que se sintetizan, en relación con el comportamiento de la sexta vocal, en los siguientes párrafos.

Con un solo informante, y en el que se consideraron solo cinco emisiones lingüísticas, Alvar y otros (1997) exploran el comportamiento espectrográfico de la denominada sexta vocal. Los autores presentan algunos datos acústicos de este segmento vocálico, esto es, valores que oscilan entre los $420 \mathrm{~Hz}$ y $460 \mathrm{~Hz}$, con un promedio de $435 \mathrm{~Hz}$ para el Fı del fono [ə], y entre $1.240 \mathrm{~Hz}$ y $1.500 \mathrm{~Hz}$, con un promedio de 1.395 $\mathrm{Hz}$ para el F2 del mismo fono. En cuanto al fono [u], los autores presentan los resultados de un solo análisis, con los valores de $360 \mathrm{~Hz}$ para el primer formante y de $1.500 \mathrm{~Hz}$ para el segundo formante.

Miotti (2005), analizó espectrográficamente las vocales de dos hablantes masculinos, de nivel socioeducacional medio-alto, pertenecientes al mapuche chileno central. En este trabajo, el autor indicó para la sexta vocal, representada como /g/, un promedio de $430 \mathrm{~Hz}$ para el F1 y un promedio de $1.545 \mathrm{~Hz}$ para el F2.

Más adelante, en un trabajo inédito, Catalán (2012) reportó datos fonético-acústicos de las vocales tónicas en informantes masculinos, de diferentes sectores geográficos, en que se habla el mapudungun. Respecto de la sexta vocal, representada como [ü], se informó un promedio de $375 \mathrm{~Hz}$ para el F1 y $1.346 \mathrm{~Hz}$ para el F2.

Por último, Sadowsky y otros (2013) realizan un análisis fonético-acústico de 9 informantes que hablan mapudungun, del sector Huapi, Novena Región, Chile. Hubo un análisis fonéticoacústico de 871 realizaciones vocálicas, en sílaba tónica o átona. Los valores determinados fueron normalizados a fin de neutralizar la variación regular que se produce en el F1 y en el F2, a consecuencia del género de los hablantes. De este trabajo, se interpretaron los triángulos vocálicos y se determinó para la sexta vocal, representada como [э], un promedio de $360 \mathrm{~Hz}$ para el F1 y un promedio de $1.480 \mathrm{~Hz}$ para el F2.

En este contexto, el trabajo que presentamos constituye un aporte relevante al conocimiento del sistema vocálico del mapudungun, en general, y de esta variante cordillerana, en particular, máxime cuando la atención en la fonética-fonología de esta lengua se ha focalizado en términos muy mayoritarios en los segmentos consonánticos

\section{Procedimientos \\ 2.1. Tipo de estudio}

Esta investigación correspondió a un abordaje descriptivo transversal, es decir, fue un estudio que presentó una etapa cuantitativa y otra cualitativa. De este modo, en una primera instancia, se analizaron espectrográficamente las producciones vocálicas y se determinaron los valores del F1 y del F2; luego, en segunda instancia, a partir de los datos fonético-acústicos cuantificados, los valores determinados se compararon con datos que se han denominado vocales referenciales, a fin de observar el comportamiento articulatorio que se deduce de los resultados de ambos formantes

\subsection{Valores de vocales referenciales}

En este trabajo, se determinó contar con valores referenciales de las vocales que se estudiaban para poder establecer las comparaciones que se planificaron realizar en el estudio. Cruttenden (2014) entrega datos del inglés británico en hablantes masculinos para [ə] tónica, indicando que el F1 presenta un valor de $513 \mathrm{~Hz}$, en tanto el F2, un valor de $1.377 \mathrm{~Hz}$. A su vez, los valores de la vocal cardinal [u], sintetizada por Delattre y otros (1952), son de $250 \mathrm{~Hz}$ para el primer formante y de $1.050 \mathrm{~Hz}$ para el segundo. 


\subsection{Selección de los informantes y mues- tra de habla}

Se utilizó la muestra de habla de chedungun, grabada en el marco del Proyecto Fondecyt № 1131095. Esta muestra se compone de la grabación de más de 100 ítems léxicos registrados a 30 sujetos, 15 del sector Queuco y 15 del sector BíoBío, hombres y mujeres, todos hablantes nativos de esta variante del mapudungun y todos también hablantes bilingües chedungun-español (de Chile). De esta muestra mayor, se seleccionó una submuestra de habla de 5 informantes masculinos del sector Queuco y 5 informantes masculinos residentes en el sector Bío-Bío.

En términos más específicos, los criterios de inclusión que se consideraron en esta investigación fueron: a) hablantes adultos (entre 30 y 60 años de edad), b) altamente competentes en el uso del mapudungun, lo que fue corroborado por un hablante nativo que pertenecía a la comunidad de mayor mantenimiento de la lengua (Butalelbun) y que a la sazón estaba a cargo de la difusión cultural en el Museo de Ralco, c) que no tuvieran impedimento físico o cognitivo incompatible con la tarea solicitada y d) respondieran afirmativamente al consentimiento informado requerido.

\subsection{Grabación de la muestra}

La muestra de habla de esta investigación fue grabada en el lugar de residencia de los pehuenches en condiciones de aislamiento acústico lo más adecuadas posible, pero que, como es de esperar, distan de las condiciones que se encuentran en un laboratorio de fonética. El instrumento utilizado, como señalamos, fue una lista léxica de más de 100 ítems; de estas palabras, se seleccionaron 20, que eran las que contenían como fonema la sexta vocal. Dado que 10 fueron los colaboradores seleccionados y que cada palabra debía ser repetida en la traducción del informante, teóricamente, 400 serían las realizaciones que ocurrirían para analizar. Sin embargo, algunas palabras no fueron respondidas y, más importante aún, no en todas ellas el acento ocurrió en la sílaba que contenía la sexta vocal.

De este modo, la cantidad de realizaciones disminuyó respecto de las ocurrencias teóricas, por lo que el total de tokens efectivamente analizados o ene muestral fue de 157.

En cuanto a la modalidad de elicitación, posterior al asentimiento del colaborador, y el cumplimiento del pentukun, por medio del colaborador mapuche pehuenche que nos apoyó, se entregó, en español, una palabra al colaborador, el cual daba como respuesta su traducción en chedungun en dos oportunidades, cada una de ellas separada por un silencio de dos segundos. Cabe hacer presente que este procedimiento, de probado rendimiento, es el que ha sido utilizado frecuentemente en las descripciones de las distintas variantes del mapudungun (Echeverría, 1964; Salas, 1978; Lagos, 1984; Álvarez-Santullano, 1986; Salamanca, 1997).

Para hacer el registro de habla, se utilizó una grabadora digital Tascam DR40. Como hemos señalado, el corpus de habla seleccionado corresponde a las vocales tónicas, pues con ello se favoreció la presencia de modo más nítido de los formantes vocálicos. Las vocales átonas no fueron analizadas, pues tienden a manifestar mucha variación no solo entre informantes, sino también en un sujeto que se esté analizando en forma particular (cfr. Soto-Barba, 2008).

\subsection{Análisis fonético-acústico}

Para realizar el análisis fonético-acústico, se seleccionó el corpus de habla de las palabras que contenían la sexta vocal y su variante. Luego se realizó una segmentación de los audios para pesquisar la palabra completa en la cual se encontraba la respectiva vocal a analizar. Una vez identificada cada una de las vocales tónicas, se procedió a medir el F1 y el F2, mediante la aplicación del programa PRAAT. La frecuencia de muestreo fue de $44 \mathrm{kHz}$ para todas las grabacio- 
nes, en tanto que para la determinación de los formantes se ajustó a $5.000 \mathrm{~Hz}$. Todos los resultados se anotaron en una tabla Excel. Además, cada enunciado se transcribió fonéticamente según el alfabeto IPA. Finalmente, se establecieron los promedios de [ə] y [u], por informante y por sector, y también se determinó el promedio general de ambos segmentos vocálicos de cada sector. Conviene dejar presente que fueron analizadas 10 realizaciones por hablantes de [ə]; sin embar-

\section{FIGURA 2}

Imagen espectrográfica de la vocal central [ə]

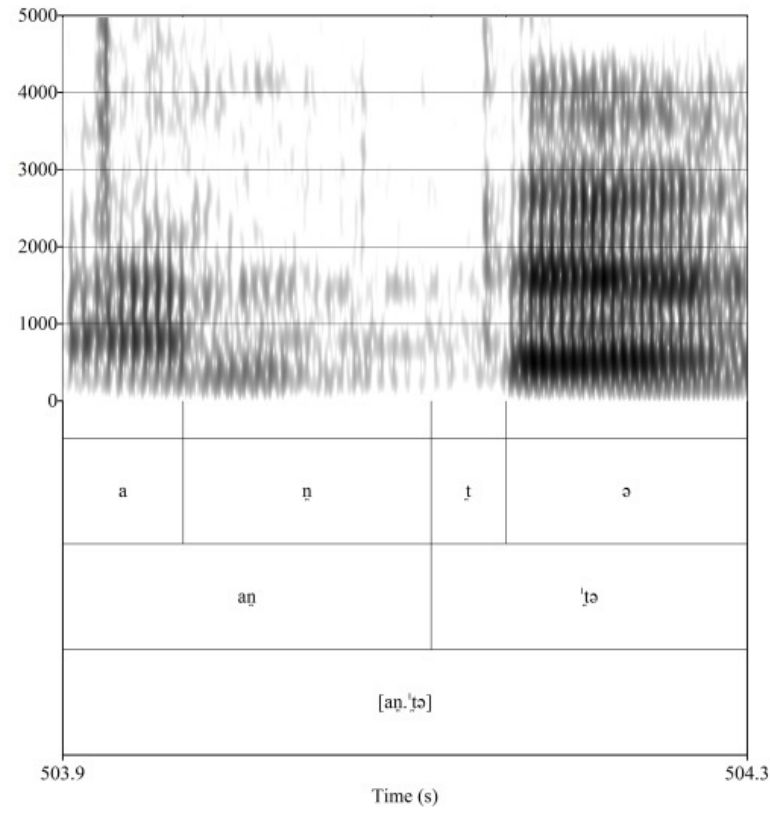

go, [u] no siempre presentó el número esperado de realizaciones

\subsection{Imágenes espectrográficas de los segmentos vocálicos [ə] y [u]}

En las figuras 2 y 3 se presenta una imagen espectrográfica de cada uno de los segmentos vocálicos observados en esta investigación.

\section{FIGURA 3}

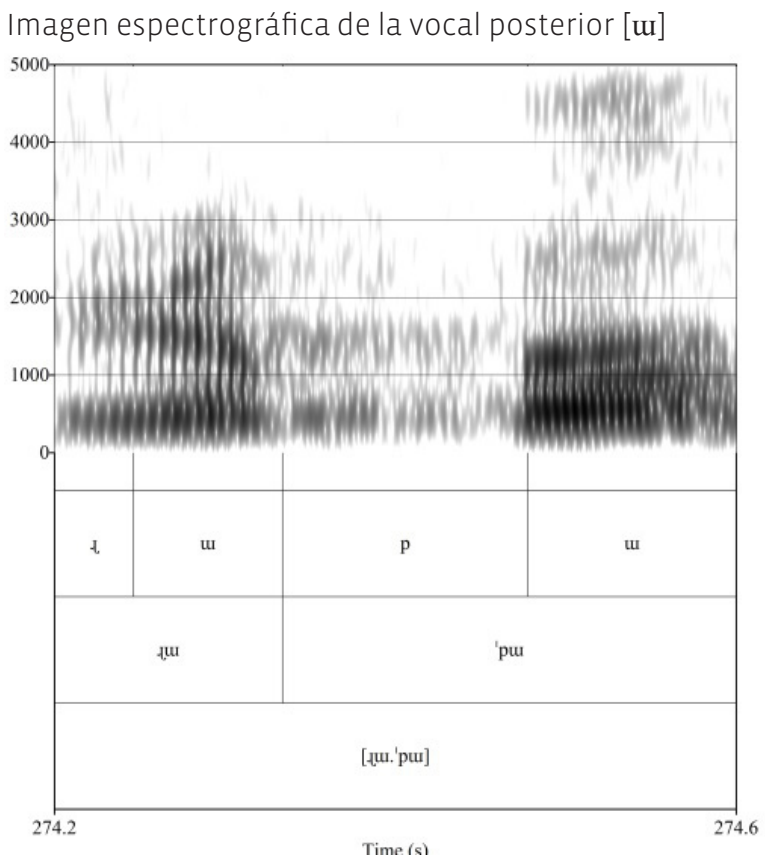

\section{Resultados}

\section{FIGURA 4}

Tabla con los valores de [ə] y [u] en los sectores Queuco y Bío-Bío, y valores referenciales

\begin{tabular}{|c|c|c|c|c|c|c|c|c|}
\hline & & & $\begin{array}{c}\text { MEDIA } \\
\text { F1 }\end{array}$ & RECUENTO & $\begin{array}{l}\text { DESVIACIÓN } \\
\text { ESTÁNDAR }\end{array}$ & $\begin{array}{c}\text { MEDIA } \\
\text { F2 }\end{array}$ & RECUENTO & $\begin{array}{l}\text { DESVIACIÓN } \\
\text { ESTÁNDAR }\end{array}$ \\
\hline \multirow{4}{*}{ Sector } & \multirow{2}{*}{ BÍO-BÍO } & [ə] & 505 & 50 & 70 & 1417 & 50 & 222 \\
\hline & & {$[\mathrm{u}]$} & 511 & 18 & 78 & 1457 & 18 & 247 \\
\hline & \multirow{2}{*}{ QUEUCO } & {$[ə]$} & 475 & 50 & 63 & 1350 & 50 & 160 \\
\hline & & & 452 & 39 & 57 & 1309 & 39 & 180 \\
\hline \multirow{2}{*}{\multicolumn{2}{|c|}{ Valor referencial }} & {$[ə]$} & 513 & \multirow{2}{*}{\multicolumn{2}{|c|}{ Sin información }} & 1377 & \multirow{2}{*}{\multicolumn{2}{|c|}{ Sin información }} \\
\hline & & {$[\mathrm{w}]$} & 250 & & & 1050 & & \\
\hline
\end{tabular}




\subsection{Medias, número de emisiones y des- viación estándar}

Tal como puede observarse en la tabla de la figura 4, se manifiestan algunas tendencias importantes relacionadas con el promedio, la cantidad de emisiones y la desviación estándar de Fı y de F2 en los sectores Queuco y Bío-Bío.

En efecto, tanto los valores promedios como los de desviación estándar muestran que el FI tiende a presentar resultados más estables en el sector Queuco que en el sector Bío-Bío, con diferencias de $30 \mathrm{~Hz}$ para [ə] y de $59 \mathrm{~Hz}$ para [u] y una diferencia de la desviación estándar de 7 puntos para [ə] y de 21 puntos para [u]. Esta relativa estabilidad, por el contrario, no se evidencia si se observan los mismos valores de F2, pues los promedios muestran diferencias de $67 \mathrm{~Hz}$ para [ə] y de $148 \mathrm{~Hz}$ para [u], en tanto se produce una notable diferencia en los valores de la desviación estándar de 62 puntos para [ə] y de 67 puntos para [u] De todas formas, lo anterior, al parecer, ocurre con alguna frecuencia cuando se comparan los valores de F1 con los valores de F2, puesto que el mismo fenómeno se pudo apreciar en las vocales de hablantes de distintos niveles socioculturales de la provincia de Ñuble (Soto-Barba, 2008).

Por otra parte, también resulta importante destacar que el número de ocurrencias mostró diferencias. Mientras todos los informantes presentaron el mismo número de realizaciones en [ə] (10 por cada sujeto, 50 en cada sector), no sucedió lo mismo con la vocal [u]], en cuya producción los hablantes del sector Queuco emitieron 39 realizaciones, en tanto que los hablantes del sector Bío-Bío apenas produjeron 18 emisiones. Este hecho muestra, en consecuencia, una mayor estabilidad de la vocal schwa en todos los pehuenches analizados y una apreciable inestabilidad de la variante de [u], sobre todo en los hablantes del sector de Bío-Bío.

\subsection{Formante 1. Vocal [ə] y vocal [u], sec- tor Queuco}

El segmento vocálico [ə] presenta un promedio más bajo $(475 \mathrm{~Hz})$ que el valor referencial $(513 \mathrm{~Hz})$, es decir, los hablantes del sector Queuco tienden a cerrar más la boca en relación con la abertura de la vocal schwa del inglés británico, con la cual se la está comparando.

En cuanto al segmento [u], se aprecia que los hablantes de chedungun de este sector tienden a abrir más la cavidad bucal si comparamos los resultados de este trabajo $(452 \mathrm{~Hz})$ con el valor referencial $(250 \mathrm{~Hz})$. Este resultado se acerca más al valor referencial de [ə] y al promedio de este segmento en los hablantes de chedungun.

\subsection{Formante 2. Vocal [ə] y vocal [u], sec- tor Queuco}

El promedio de [ə], con un valor de $1.350 \mathrm{~Hz}$, es bastante similar al valor referencial, es decir, 1.377 Hz, hecho que indicaría una cierta estabilidad de este segmento de acuerdo con lo esperado, vale decir, en el chedungun, esta vocal tiende a articularse llevando la lengua hacia el centro de la boca.

Una situación distinta se aprecia con el segmento vocálico [u]], pues el promedio de $1.309 \mathrm{~Hz}$ se aleja bastante del valor referencial de 1.050 $\mathrm{Hz}$ e indica que este fono se comporta más bien como la vocal [ə] en el chedungun del sector Queuco. Así, los resultados de esta zona muestran que a este sonido no se lo podría catalogar como una vocal con tendencia a articularse llevando la lengua hacia la zona posterior o velar de la cavidad bucal, sino más bien con tendencia a llevar la lengua hacia el centro de la boca.

\subsection{Formante 1. Vocal [ə] y vocal [u], sec- tor Bío-Bío}

El segmento vocálico [ə] del sector Bío-Bío presenta un promedio levemente más bajo (505 $\mathrm{Hz}$ ) que el valor referencial $(513 \mathrm{~Hz})$, esto es, los hablantes del sector Bío-Bío tienden a articular 
esta vocal de manera muy similar a la forma de articular este sonido del inglés británico, vale decir, con tendencia a abrir la boca como en un segmento vocálico con bastante cercanía a una vocal abierta.

En relación con el segmento [u], los resultados indican que los hablantes de chedungun del sector Bío-Bío tienden a abrir más la cavidad bucal, si comparamos los promedios de este trabajo $(511 \mathrm{~Hz})$ con el valor referencial $(250 \mathrm{~Hz})$. Este resultado se acerca todavía más al promedio de la sexta vocal [ə], en los hablantes de chedungun, y es prácticamente idéntico al valor referencial del mismo sonido.

\subsection{Formante 2. Vocal [ə] y vocal [u], sec- tor Bío-Bío}

En cuanto a [ə], el promedio de esta vocal (1.417 $\mathrm{Hz}$ ) es bastante similar al valor referencial (1.377 $\mathrm{Hz}$ ), hecho que indicaría una cierta estabilidad de este fonema de acuerdo con lo esperado, es decir, con una producción en que la lengua se ubica hacia el centro de la boca.

Una situación diferente se aprecia con el fono [u], puesto que el promedio del segmento $(1.457 \mathrm{~Hz})$ en esta investigación evidencia que este sonido se comporta de manera muy similar a lo que ocurre con la vocal [ə], alejándose notablemente del valor referencial $(1.050 \mathrm{~Hz})$. Tal indicio acústico permite colegir que este sonido tampoco se podría caracterizar como vocal pos terior o velar, así como también se aprecia que este fenómeno se comporta de manera muy similar a lo observado en el sector Queuco.

\subsection{Comparación de resultados del F1 y del F2, sectores Queuco y Bío-Bío (F1 y F2)}

\subsubsection{Formante 1}

Con la vocal [ə], ambos sectores tienden a cerrar más la boca de acuerdo con el valor referencial definido, que corresponde a $513 \mathrm{~Hz}$, fenómeno más acentuado en el sector Queuco respecto del sector Bío-Bío (475 Hz vs 505 Hz).

Con la variante $[\mathrm{u}]$, la situación observada resulta sorprendente, pues, en ambos sectores, la tendencia es a abrir bastante más la boca según el valor de referencia definido de $250 \mathrm{~Hz}$, si bien es el sector de Bío-Bío, otra vez, el que lo hace de modo más notable $(511 \mathrm{~Hz}$ vs $452 \mathrm{~Hz})$. Tal como se indicó con anterioridad, estos resultados se acercan mucho más al Fı de la vocal [ə].

\subsubsection{Formante 2}

Con la vocal [ə], se aprecia que, en ambos sectores, los promedios se acercan al valor referencial definido (1.377 Hz). Aún así, el sector de Queuco tiende a llevar la lengua hacia una zona menos anterior a la esperada $(1.350 \mathrm{~Hz})$, en tanto que el sector Bío-Bío, al contrario, tiende a llevar la lengua hacia una zona más anterior a la esperada (1.417 Hz).

Respecto del sonido [u], en ambos sectores, los promedios se alejan notablemente del valor referencial esperado, que corresponde a $1.050 \mathrm{~Hz}$, es decir, los hablantes de ambos grupos llevan la lengua hacia la zona del centro de la boca, aunque es el sector de Bío-Bío el que tiende a acentuar más esta articulación respecto del sector Queuco (1.457 Hz vs. 1309 $\mathrm{Hz}$ ). Estos hechos, además, demuestran una fuerte inclinación a producir esta vocal como [ə] en el chedungun de Alto Bío-Bío, si se tiene en cuenta el valor referencial de la vocal schwa de $1.377 \mathrm{~Hz}$.

\subsection{Comparación de los resultados de- terminados en el chedungun vs. otros estudios del mapudungun (F1 y F2)}




\section{FIGURA 5}

Tabla con los valores de [ə] y [u] en los sectores Queuco, Bío-Bío y valores de los trabajos de Alvar y otros (1997), Miotti (2005), Catalán (2012) y Sadowsky y otros (2013)

\begin{tabular}{|c|c|c|c|c|c|c|c|c|c|c|c|c|}
\hline & \multicolumn{2}{|c|}{ QUEUCO } & \multicolumn{2}{|c|}{ Bío-Bío } & \multicolumn{2}{|c|}{$\begin{array}{l}\text { ALVAR } \\
\text { Y OTROS }\end{array}$} & \multicolumn{2}{|c|}{ MIOTTI } & \multicolumn{2}{|c|}{ CATALÁN } & \multicolumn{2}{|c|}{$\begin{array}{l}\text { SADOWSKY } \\
\text { Y OTROS }\end{array}$} \\
\hline & [ə] & {$[\mathrm{w}]$} & [ə] & {$[\mathrm{w}]$} & [ə] & {$[\mathrm{w}]$} & /g/ & {$[\mathrm{w}]$} & /ü/ & {$[\mathrm{w}]$} & [э] & [u] \\
\hline $\mathrm{F}_{1}$ & 475 & 452 & 505 & 511 & 435 & 360 & 430 & -- & 375 & --- & 360 & --- \\
\hline $\mathrm{F}_{2}$ & 1350 & 1309 & 1417 & 1457 & 1395 & 1500 & 1545 & -- & 1346 & -- & 1480 & -- \\
\hline
\end{tabular}

\subsubsection{Chedungun vs. Alvar y otros (1997) (F1 y F2)}

En relación con el trabajo de Alvar y otros (1997), con [ə], si se comparan los promedios del F1, esta investigación se aleja de esos resultados, y si se comparan los promedios del F2, se acercan.

En efecto, en cuanto al F1, estos autores establecieron un promedio de $435 \mathrm{~Hz}$ para [ə], es decir, un valor que demuestra una articulación en que la cavidad oral tiende a cerrarse, en tanto que en esta investigación el promedio de [ə] fue de $475 \mathrm{~Hz}$ para el sector Queuco y de $505 \mathrm{~Hz}$ para el sector Bío-Bío, vale decir, valores que muestran tendencia a articular esta vocal con la boca un tanto más abierta.

En cuanto al F2, los resultados son más cercanos, pues el promedio del estudio citado fue de $1.395 \mathrm{~Hz}$ y los de este trabajo fueron de $1.350 \mathrm{~Hz}$ y 1.417 Hz para Queuco y Bío-Bío, respectivamente; vale decir, los resultados de Alvar y otros (1997) se ubican entre el sector de Queuco y de Bío-Bío observados en este estudio, y demuestran que en todos los casos la tendencia fue a llevar la lengua hacia el centro de la cavidad bucal.

Con [u] , el comportamiento fue diferente en relación con los promedios de Fı y relativamente más cercano en relación con los promedios de F2.

Así, en el trabajo citado, el promedio de FI fue de $360 \mathrm{~Hz}$, es decir, el hablante tendía a cerrar la boca, mientras que, ya sea en Queuco o Bío-Bío, la tendencia fue a abrir más la boca, si se considera que los promedios en estos sectores fueron $452 \mathrm{~Hz}$ y $511 \mathrm{~Hz}$, respectivamente. En otras pala- bras, mientras Alvar y otros (1997), efectivamente, constataron una [u] cerrada, los hablantes de chedungun tendieron a pronunciarla con la boca más abierta, como si fuese una schwa.

En cuanto al comportamiento del F2, la situación es distinta, pues el promedio del trabajo citado fue de $1.500 \mathrm{~Hz}$, vale decir, un valor más cercano a la vocal [a], y los de Queuco y Bío-Bío fueron de $1.309 \mathrm{~Hz}$ y de $1.457 \mathrm{~Hz}$, respectivamente. Dicho de otro modo, el hablante del estudio de Alvar y otros (1997) tendió a llevar la lengua hacia el centro de la boca, en tanto los hablantes de Queuco y Bío-Bío tendieron a llevar la lengua un poco más hacia la zona posterior del pabellón bucal, nuevamente como si fuese una schwa.

\subsubsection{Chedungun vs. Miotti (2005)}

En relación con el trabajo de Miotti (2005), si se comparan los promedios del F1, esta investigación se aleja de esos resultados. En efecto, en cuanto al F1, este autor estableció un promedio de $430 \mathrm{~Hz}$ para [ə], es decir, un valor que demuestra una articulación en que la cavidad oral tiende a cerrarse, en tanto que en esta investigación el promedio de [ə] fue de $475 \mathrm{~Hz}$ para el sector Queuco y de $505 \mathrm{~Hz}$ para el sector Bío-Bío, vale decir, valores que muestran tendencia a articular esta vocal con la boca un tanto más abierta.

En cuanto al comportamiento del F2 de [ə], si se comparan los promedios de esta investigación con los determinados por Miotti (2005), los resultados también se alejan, pues, en el trabajo alu- 
dido, el F2 alcanzó $1.545 \mathrm{~Hz}$, en tanto que en este estudio, en el sector Queuco, el F2 fue de $1.350 \mathrm{~Hz}$ y, en el sector Bío-Bío, fue de 1.417 Hz. En otras palabras, mientras los hablantes de Miotti tendían a articular esta vocal con la lengua ubicada hacia el centro de la boca, como ocurre con la vocal [a], los hablantes de chedungun tendían a pronunciar este segmento llevando la lengua un poco más hacia la zona posterior de la cavidad bucal, como ocurre con la vocal [ə].

Dado que el estudio de Miotti (2005) no tuvo entre sus objetivos caracterizar variantes fonéticas de la sexta vocal, no fue posible establecer una comparación del comportamiento de Fr y de F2 con los resultados establecidos para [u] en este trabajo.

\subsubsection{Chedungun vs. Catalán (2012)}

Con el Fı de la vocal/ü/, los resultados indicaron que los hablantes observados por Catalán (2012) tendieron a articularla con la boca más bien cerrada, en tanto los hablantes de chedungun tendieron a abrir un poco más la boca, a tal punto que esta vocal fue producida no como [ü], sino más bien como [ə]. Ello se corrobora al contrastar los promedios: $375 \mathrm{~Hz}$ para/ü/ vs. $490 \mathrm{~Hz}$ para [ə].

Por su parte, el F2 de la vocal [ə] presentó, en ambas investigaciones, resultados muy similares, esto es, $1.346 \mathrm{~Hz}$ para /ü/ vs. $1.383 \mathrm{~Hz}$ para [ə], fenómeno de características fonético-fonológicas similares más allá de la representación fonológica o fonética que se utilizó en uno u otro trabajo para representar este segmento vocálico.

Dado que el estudio de Catalán (2012) no tuvo, necesariamente, entre sus objetivos caracterizar variantes fonéticas de la sexta vocal, tampoco fue posible establecer una comparación del comportamiento de F1 y de F2 con los resultados establecidos para [u] en este trabajo.

\subsubsection{Chedungun vs. Sadowsky y otros (2013)}

En cuanto al F1, con la vocal [ə], en relación con la vocal equivalente del trabajo aludido, esto es [э], los resultados indicaron que los hablantes observados por Sadowsky y otros (2013) mostraron tendencia a articularla con la boca más bien cerrada, en tanto los hablantes de chedungun de ambos sectores tendieron a abrir un poco más la boca. Ello se corrobora al contrastar los promedios: en el mapudungun hablado en Huapi, 360 $\mathrm{Hz}$; en el chedungun, $475 \mathrm{~Hz}$ en el sector Queuco y 505 Hz en el sector Bío-Bío.

En relación con el F2 de esta vocal, el trabajo de Sadowsky y otros (2013) presentó promedios más cercanos, vale decir, $1.480 \mathrm{~Hz}$ para [o] y 1.350 Hz en el sector Queuco y 1.417 Hz en el sector BíoBío. De todas formas, la investigación aludida, de acuerdo con los promedios observados, indica que los hablantes de Huapi se tienden a llevar la lengua un poco más al centro de la boca que lo producido en el chedungun, dialecto en el que la lengua se retrasa levemente hacia la zona posterior de la cavidad bucal.

Si bien el estudio de Sadowsky y otros (2013) propone una co-variante de la sexta vocal [э], es decir [i] ción que se produce en vocales átonas, por lo que no fue posible realizar una comparación de los formantes del estudio citado con el F1 y el F2 que se determinaron en [u], en esta investigación.

\section{Conclusiones}

El análisis fonético-acústico de los segmentos vocálicos en estudio demuestra que, en general, los hablantes del sector Queuco y los hablantes del sector Bío-Bío tienden a articular estos sonidos de manera similar. Dicho de otra manera, el chedungun, en cuanto a la pronunciación de estas vocales tónicas, funciona como un solo dialecto del mapudungun.

Todas las realizaciones de [ə], ya sea según los promedios del primer formante, o bien según los del segundo formante, efectivamente, se produjeron como una vocal schwa. Algo distinto ocu- 
rrió en el caso de [u], pues los resultados del Fıy del F2 de este trabajo indican, por una parte, que varias realizaciones esperadas como vocal posterior no redondeada fueron articuladas como otra vocal del chedungun (principalmente [u]) y, por otra, que, salvo casos muy excepcionales, la mayoría de las ocurrencias esperadas como [u], finalmente, fueron producidas como schwa.

En este sentido, la contundencia de los resultados establecidos en este trabajo permite afirmar que, al menos en las vocales tónicas del chedungun pronunciadas por hablantes de sexo masculino, la forma básica de la sexta vocal en este dialecto del mapudungun es /ə/. Junto con ello, a la luz de los datos que entreguen nuevas pesquisas con los frentes no cubiertos aquí, también convendría revisar la notación correspondiente a su(s) alófono(s) prominente(s), pues, de no mediar hallazgos muy distintos a los aquí presentados, cabría una notación modificada, distinta o complementaria para [u]. Hay que recordar, en este sentido, que en este artículo los datos fueron cotejados con este segmento alto posterior no redondeado (representado habitualmente [ü] o [it]), pues es el más recurrente en las presentaciones de la alofonía de la sexta vocal en los estudios históricos del mapudungun, después de [ə].

Por otra parte, no es descartable que las diferencias producidas al comparar los datos con otros estudios sean atribuibles a características dialectales, pues, si bien el pan-mapudungun ha sido reportado con una importante homogeneidad, hay aspectos fónicos que claramente establecen diferencias diatópicas, como la sonoridad de las consonantes fricativas labiodental e interdental. Y, por cierto, tampoco es descartable la influencia que el español tuvo y tiene en esta lengua/variante, la que se ha acrecentado a partir del último cuarto de siglo.

Resulta conveniente recalcar, entonces, que las tendencias aquí presentadas deben ser corroboradas con nuevos trabajos fonético-acústicos en los cuales se consideren otras variables, como, por ejemplo, el carácter átono de la sílaba de los enunciados elicitados o la consideración de ha- blantes femeninos que también conforman la muestra mayor de habla de la investigación del chedungun de Alto Bío-Bío.

\section{Bibliografía citada}

Alvar, Manuel, Francisco Moreno e Hiroto Ueda, 1997: "La sexta vocal del mapuche", Thesaurus LII, 183-189.

Álvarez-Santullano, Pilar, 1986: "Descripción fonemática del Huilliche: estudio comparativo", Alpha 2, 45-50

Augusta, Félix José de, 1903: Gramática Araucana, Valdivia: Imprenta Central J. Lampert.

Catalán, Rosa, 2012: Estudio fonético acústico de las vocales del mapudungun. Tesis de Magíster en Letras con Mención en Lingüística. Pontificia Universidad Católica de Chile.

Contreras, Heles y Max Echeverría, 1965: "Araucanian Phonemics", IJAL 31(2), 132-135.

Cruttenden, Alan, 2014: Gimson's pronunciation of English, Londres: Routledge.

Dehnhardt, Macarena, Adrián Valenzuela y Natalia Villarroel, 2015: Adaptación de la sexta vocal/ü/ del mapudungun al español de Chile. Una revisión del Diccionario Etimolójico de Rodolfo Lenz. Tesis de Licenciatura en Lengua y Literatura Hispánica mención Lingüística. Universidad de Chile.

Delattre, Pierre y otros, 1952: "An experimental study of the acoustic determinants of vowel color; observations on one- and two-formant vowels synthesized from spectrographic patterns", Word 8, 195-210.

EcheverRía, Max, 1964: "Descripción fonológica del mapuche actual", Boletín del Instituto de Filología de la Universidad de Chile XVI, 13-59. 
Febrés, Andrés, 1765: Gramática Araucana, o sea, Arte de la Lengua General de los Indios de Chile, Buenos Aires: Impreso por Juan Alsina en 1884.

HAvestAdt, Bernardo, 1777: Chilidúgú sive Res Chilenses vel Descriptio Status tum Naturalis, tum Civilis, cum Moralis Regni Populique Chilensis, inserta suis locis perfectae ad Chilensem Linguam Manudictioni, Deo O.M [...] opera [...] Bernardi Havestadt. Monasterii Westphaliae Typis Aschendorfianis. Tomus I, Tomus II, Tomus III.

Henriquez, Marisol y Gastón Salamanca, 2012: "Rasgos prominentes de la fonología segmental del chedungun hablado por los escolares del Alto Bío-Bío", Alpha 34, 153-171.

LaGos, Daniel, 1984: "Fonología del mapuche hablado en Victoria”, Actas Jornadas de Lengua y Literatura Mapuche, 41-50.

LAGos, Daniel, 2001: Fonología de las lenguas indígenas en Chile, Valparaíso: Celto, Universidad de Playa Ancha.

Lenz, Rodolfo, 1895-1897: Estudios Araucanos [son doce artículos, numerados del I al XII, aparecidos en Anales de la Universidad de Chile. Tomos XCXCVIII].

Mıтt, Renzo, 2005: "Analisi acustico-uditiva del vocalismo mapuche. Aspetti temporale e timbrico", R.L.A. 43(1), 45-64.

SADowsky, Scott y otros, 2013: "Mapudungun", Journal of the International Phonetic Association 43(1), 87-96.

Salamanca, Gastón, 1997: "Fonología del pehuenche hablado en el Alto Bío-Bío”, RLA 35, 113-124.

Salamanca, Gastón y otros, 2009: "Mapuche hablado en Melipeuco: Fonemas segmentales, fonotaxis y comparación con otras variedades", Logos: Revista de Lingüística, Filosofía y Literatura 19(2), 74-95.
SalAs, Adalberto, 1978: "Mapuche-Español. Análisis fonológico contrastivo", VICUS Cuadernos Lingüística de la Universidad Católica de Temuco II, 57-85.

Salas, Adalberto,1992: El mapuche o araucano, Madrid: Mapfre.

SÁnchez, Gilberto, 1989: "Relatos orales en pewenche chileno", Anales de la Universidad de Chile. Estudios en honor de Yolando Pino Saavedra 17, 289-360.

Smeets, Ineke, 2008: A Mapuche Grammar, BerlínNew York: Mouton Grammar Library.

Soto-BARBA, Jaime, 2008: Variación fonética consonántica y vocálica en el habla urbana y rural de la provincia de Nuble. Tesis de doctorado, Universidad de Valladolid.

SuÁrez, Jorge, 1959: "The phonemes of an Araucanian dialect”, IJAL 25, 77-181.

VALDIVIA, Luis de, 1887[1606]: Arte y gramática general de la lengua que recorre en todo el Reyno de Chile, con un Vocabulario y Confessonario, Lima: Francisco del Canto. [Edición facsimilar de Julio Platzmann].

Zúñiga, Fernando, 2006: Mapudungun, el habla mapuche, Santiago: Centro de Estudios Públicos. 